\title{
Cutaneous Metastases in a Patient With Muir-Torre Syndrome
}

Michael Kunz, MD; Omar Bari, MD²; Ahmed K. Alomari, MD³; Ally-Khan Somani, MD, $\mathrm{PhD}^{1}$

1. Division of Dermatologic Surgery and Cutaneous Oncology Department of Dermatology Indiana University School of Medicine Indianapolis, Indiana

2. Division of Dermatology University of California Los Angeles School of Medicine Los Angeles, California Department of Dermatology Indiana University School of Medicine Indianapolis, Indiana

3. Department of Pathology Indiana University School of Medicine Indianapolis, Indiana 
We describe the clinical features of a man with Muir- Torre syndrome (MTS) who was recently diagnosed with gastric adenocarcinoma and then presented to the Mohs micrographic surgery (MMS) unit for treatment of chin and neck lesions. Final pathology confirmed that both lesions were gastric adenocarcinoma cutaneous metastases; we report the first case of cutaneous metastasis from gastric adenocarcinoma in a patient with MTS. Cutaneous metastases are rare and account for just $2 \%$ of all skin cancers. They generally manifest as dermal nodules, although morphology may vary. Cutaneous metastases are often found overlying the visceral malignancy, but they may also appear at distant locations. The most common cancers associated with cutaneous metastases include breast, colon, lung, and melanoma. ${ }^{1}$ A 46-year-old Caucasian man with MTS presented to the MMS unit for treatment of an erythematous nodule on the chin and an erythematous nodule on the posterior neck. He had a left pubic sebaceous carcinoma excised 2 years ago and a strong family history of malignancy. Three months before MMS, the patient had been diagnosed with metastatic gastric adenocarcinoma by gastroenterology. After his first cycle of chemotherapy, the patient alerted his oncologist about a tender cystic nodule on the chin that had been present for several months. The oncologist performed fine needle aspiration. Cytopathologic examination was consistent with malignant epithelial cells (Figure 1); the differential diagnosis included sebaceous carcinoma and gastric adenocarcinoma cutaneous metastasis. A referral to dermatology was subsequently made. Cutaneous examination of the chin revealed a $1.5-\mathrm{cm}$ erythematous nodule (Figure $2 \mathrm{~A}$ ). In addition, a $1-\mathrm{cm}$ nontender erythematous nodule was noted on the left posterior neck (Figure 2B); the patient stated that the latter lesion had been present for many weeks. The clinical differential diagnosis included cutaneous metastasis, epidermal inclusion cyst, and sebaceous carcinoma. The patient was referred to the MMS unit for surgical treatment. Excisional biopsy was performed on the neck nodule, whereas MMS was conducted on the chin nodule. The tumor-positive margins on the first MMS layer showed a poorly differentiated carcinoma consisting of basaloid cells (Figure 3). The tumor was completely cleared after 2 additional MMS layers. Reconstruction was then performed with a complex linear closure. Debulked and nonmarginal MMS tissue blocks from the chin and the excised lesion from the neck were sent for permanent pathology (formalin-fixed and paraffin embedded) to clarify the final diagnosis. On permanent sections, both specimens appeared identical: poorly differentiated malignant neoplasm with glandular structures, signet ring cell appearance, and "dirty" necrosis with highly pleomorphic cells were seen (Figure 4). Malignant cells were weakly positive for CK7 and weakly and focally positive for CDX-2. P63, androgen receptor, CK20, and D2-40 stains were negative. Based on this morphology and immunohistochemical profile, the carcinoma appeared consistent with metastasis from the patient's known gastric adenocarcinoma. The patient returned to the MMS unit for 2week postoperative follow-up. Both scars were found to be healing well, and he was referred back to oncology. After 7 cycles of chemotherapy, the patient was transitioned to pembrolizumab. Subsequent diagnostic laparoscopy suggested complete response to immunotherapy. 
Figure 1. Low $(A, x 200)$ and high $(B, x 600)$ power views of the fine needle aspiration biopsy from the chin nodule (direct smear with Diff-Quick stain) show numerous loosely cohesive groups of atypical, frankly malignant epithelial cells.

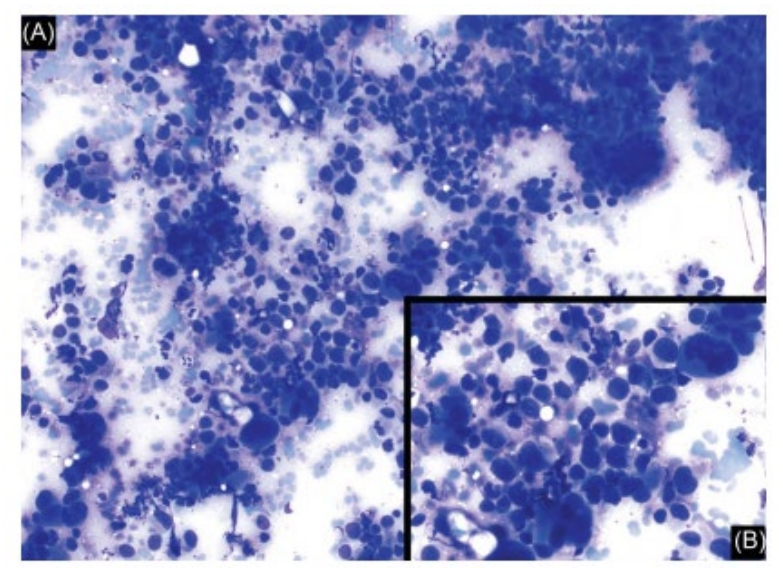

Figure 2. (A) Solitary erythematous nodule is seen on the chin (A), whereas a small erythematous nodule is evident on the left posterior neck (B).

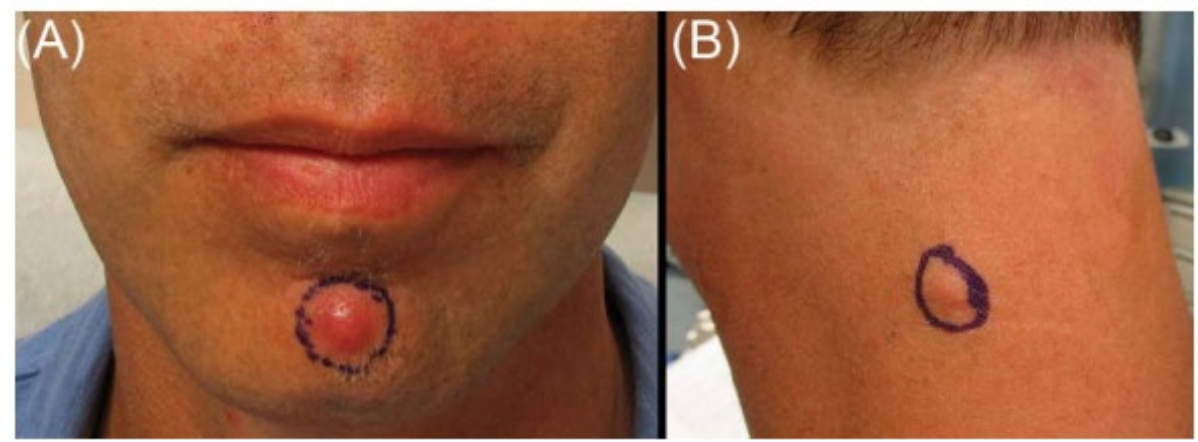

Figure 3. The first Mohs micrographic surgery layer supports cutaneous metastasis by showing infiltrative strands of poorly differentiated carcinoma cells disconnected from the epidermis ( $A$ : frozen section, H\&E original magnification x20). Higher magnification reveals poorly differentiated neoplasm with nuclear polymorphism (B: frozen section, $H \& E$ original magnification $\times 200$ ).

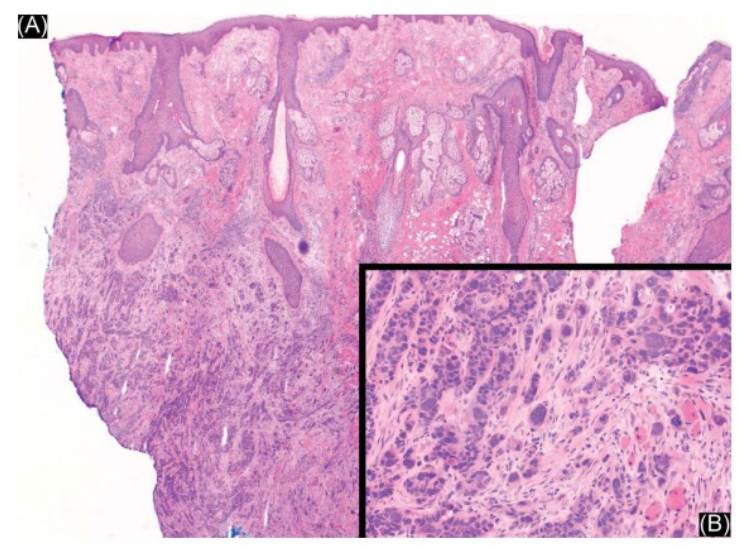


Figure 4. Formalin-fixed, paraffin-embedded section from the chin nodule confirms cutaneous metastasis by displaying a poorly differentiated malignant carcinoma with glandular structures lacking epidermal connection (A: H\&E original magnification x40). Higher magnification displays dirty necrosis (blue arrow) and signet ring cells (black arrows) (B: H\&E original magnification $\mathrm{x} 400$ )

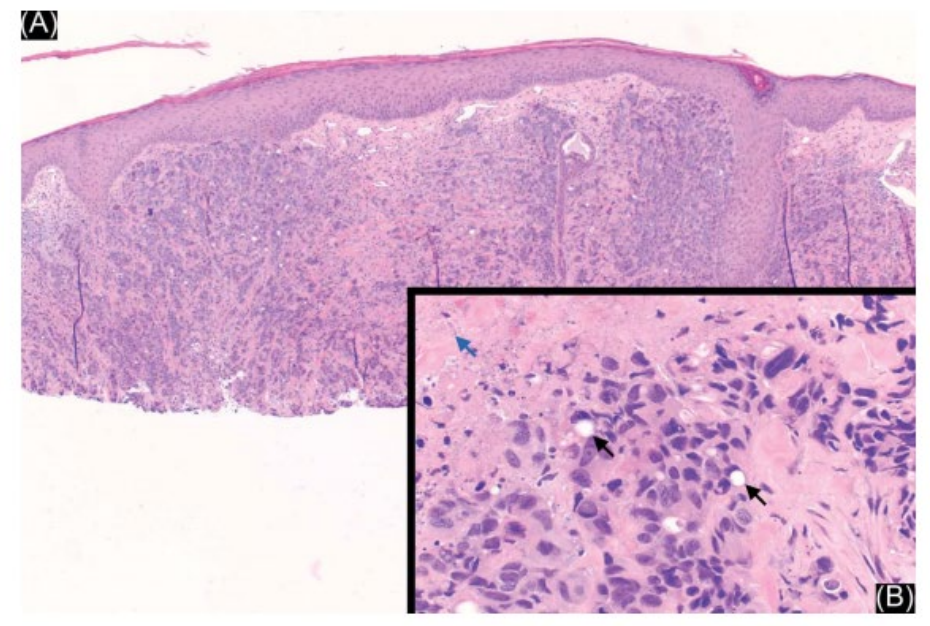

\section{DISCUSSION}

Muir-Torre syndrome is a variant of hereditary nonpolyposis colorectal cancer (HNPCC) syndrome, which is also known as Lynch syndrome. Approximately $9 \%$ of patients with known HNPCC are affected by MTS; this diagnosis should be clinically suspected when patients develop at least one sebaceous neoplasm before the age of 60 years in the context of personal or family history of Lynch syndrome-related cancers. Sebaceous neoplasms seen in MTS include adenoma, carcinoma, and sebaceoma, although MTS patients also develop multiple keratoacanthomas. MTS I, which represents $65 \%$ of cases, is inherited in an autosomal dominant fashion. The diagnosis of MTS I is supported by abnormal mismatch repair (MMR) protein staining on immunohistochemistry and microsatellite instability by genetic testing. By comparison, the autosomal recessive MTS II should be considered in patients in whom no MMR defects are found.2 The most common internal malignancy in MTS is colorectal adenocarcinoma. Other reported malignancies include cancers of the brain, breast, endometrium, noncolonic gastrointestinal tract, lung, ovary, pancreas, and uroepithelial tract.2 Metastatic disease is not uncommon in MTS; in an analysis of 52 MTS patients with internal malignancies, $60 \%$ of patients developed metastases to various sites. 3 However, to the best of our knowledge, there has only been 1 previously reported case of cutaneous metastasis in a patient with MTS; the previous case was a 41-year-old woman who developed a facial cutaneous metastasis from mucinous hepatic cholangiocarcinoma. Although our patient demonstrated loss of expression of mutator $L$ homologue (MLH) 1 , the other patient had a genetic mutation in mutator $\mathrm{S}$ homologue $(\mathrm{MSH}) 2.4$ Cutaneous metastasis has been reported to occur in only $0.8 \%$ of all gastric cancers and in $3 \%$ of colorectal cancers. 5 Cutaneous metastases may arise from local spread to overlying skin, although more 
distant spread is likely through blood vessels or dermal lymphatics. Prompt recognition of cutaneous metastases may decrease morbidity and mortality. ${ }^{5}$

Dermatologic surgeons should consider the possibility of cutaneous metastasis in patients with MTS. Shave or punch biopsies are the preferred method to confirm histological diagnosis and should be sought for definite diagnostic confirmation of malignant epithelial cells found by fine-needle aspiration biopsy before referral for MMS. However, if malignant lesions involve cosmetically sensitive facial areas, are symptomatic, and contribute to the patient's anxiety, then complete removal of the lesions through MMS can be considered. By performing MMS directly, patients avoid a second procedure and limit a delay in diagnosis. If MMS is performed in this context, the debulked tumor should be sent to pathology to clearly define the malignant lesions. The diagnosis of cutaneous metastases should prompt a thorough review of systems, a symptom guided initial laboratory work-up, and immediate referral to an oncologist for cancer staging. Further referrals of patients with MTS and cutaneous metastases should include gastroenterology and dermatology. Gastroenterologists will offer screening colonoscopies and upper endoscopies, whereas dermatologists should conduct annual full-body skin examinations to monitor for sebaceous carcinomas, keratoacanthomas, and cutaneous metastasis.

\section{CONCLUSION}

We report the first case of cutaneous metastasis from gastric adenocarcinoma in a patient with MTS. Our patient arrived at the MMS unit without a definitive diagnosis; thus, the preoperative pretest probability for sebaceous carcinoma was high given the known propensity of patients with MTS to develop sebaceous neoplasms. Because cutaneous metastases may have variable morphology and distant sites of presentation, the differential diagnosis for new lesions in patients with MTS should include cutaneous metastasis.

\section{REFERENCES}

1. Alcaraz I, Cerroni L, Rütten $A$, Kutzner $H$, et al. Cutaneous metastases from internal malignancies: a clinicopathologic and immunohistochemical review. Am J Dermatopathol 2012;34:347-93.

2. John AM, Schwartz RA. Muir-Torre syndrome (MTS): an update and approach to diagnosis and management. J Am Acad Dermatol 2016;74:558-66.

3. Cohen PR, Kohn SR, Kurzrock R. Association of sebaceous gland tumors and internal malignancy: the Muir-Torre syndrome. Am J Med 1991;90:606-13.

4. Vernez M, Hutter $P$, Monnerat $C$, Halkic $N$, et al. A case of MuirTorre syndrome associated with mucinous hepatic cholangiocarcinoma and a novel germline mutation of the MSH2 gene. Fam Cancer 2007;6:141-5. 
5. Hu SC, Chen GS, Wu CS, Chai CY, et al. Rates of cutaneous metastases from different internal malignancies: experience from a Taiwanese medical center. J Am Acad Dermatol 2009;60:379-87. 